\title{
Signal Processing for Estimating Energy Expenditure of Elite Athletes using Triaxial Accelerometers
}

\author{
Andrew Wixted, David Thiel, Daniel James \\ Center for Wireless Monitoring and Applications \\ CRC for Microtechnology \\ Griffith University, Australia \\ a.wixted@griffith.edu.au
}

\author{
Allan Hahn, Christopher Gore, David Pyne \\ Department of Physiology \\ Australian Institute of Sport, Canberra, Australia
}

\begin{abstract}
Fitness development of elite athletes requires an understanding of physiological factors such as athlete energy expenditure (EE). For athletes involved in football at the elite level, it is necessary to understand the energy demands during competition to develop training regimes. By identifying an appropriate $\mathrm{EE}$ estimator in triaxial accelerometer data, in conjunction with identifying sources of inter-athlete variance in that estimator, signal processing was developed to extract the estimator. In this system, low-power signal processing was implemented to extract both the EE estimator and other information of physiological and statistical interest.
\end{abstract}

\section{INTRODUCTION}

Sports scientists monitoring the EE of elite athletes during training rely predominately on athlete self-monitoring augmented by monitored training sessions using traditional physiological measures such as heart rate. Self-monitoring relies on the athlete recording their activities in a diary [1] and using a reference table to estimate EE [2]. Estimating athlete EE during competition in field sports can be labor intensive and involves video monitoring and manual activity categorization. Micro-Electro Mechanical Systems (MEMS), particularly accelerometers provide an alternative, noninvasive sensor for use in automatic activity monitoring.

The purpose of this study was to establish methods for estimating the EE of football players in competition and training. Accelerometers were chosen as the sensor because they are unobtrusive and can provide information pertaining to various activities of interest such as walking, running, athlete orientation and impact contacts between athletes.

In most football codes, there is a high intensity of activity in the general proximity of the ball, although for the majority of the game time, most players are involved in relatively low intensity activity. This low intensity activity is interspersed with short bursts of high intensity activity followed by a recovery interval of variable duration. The predominant activities of interest are: stationary, walking, running, sprinting, contacts (tackles, hit-ups and other challenges). For physiological monitoring, the activity intensity, duration and temporal relationship to other activities are important determinants of the overall physical load.

This research sponsored by the Australian Sports Commission and the CRC for Microtechnology, Hawthorne, Victoria, Australia.
Due to the nature of competitive football, any monitoring device must be as small and unobtrusive as possible. With the current state of microelectronics and MEMS, the limiting factor on size and mass is the power source. By interpreting the biomechanical activity as it relates to estimated EE, appropriate low-power signal processing algorithms have been developed. Simplified signal processing has reduced the processor load enabling a reduction in battery size.

\section{A. Accelerometers as Energy Estimators}

Montoye et. al. [3] (1983) developed a small portable uniaxial accelerometer and undertook a comparison between the accelerometer, volume of oxygen $\left(\mathrm{VO}_{2}\right)$ consumed and mercury switches. Subjects randomly worked through a series of physical activities chosen for their similarity to daily activities. The accelerometer output proved to be highly reproducible and, across the combined activities had moderate to high individual and group correlations with $\mathrm{VO}_{2}$ $(\mathrm{n}=21$, individual $\mathrm{r}=0.63-0.89$, mean $\mathrm{r}=0.79$, group $\mathrm{r}=0.74)$.

Commercial accelerometer based devices became available in the 1990 's, subsequently the vertically mounted uniaxial accelerometer, and more recently the triaxial accelerometer, have become widely used in studies of EE in free-living subjects [4].

While various accelerometry devices are available and group results may exhibit correlation with $\mathrm{EE}$, individual results can vary widely. For this research, it was originally hypothesized that the energy efficiency of a runner's technique may be discernable in the kinematic data from a triaxial accelerometer platform. Through experiments and analysis, we sought to establish whether variations in technique are responsible for the inter-subject variability shown in the acceleration integral.

\section{EXPERIMENTAL DESIGN}

Two separate treadmill studies were conducted in this project. First, ten male Australian Football players walked and ran using their natural cadence at ten speeds on a motorized treadmill. These athletes were monitored by a triaxial accelerometer system fixed into a semi-elastic belt, 
fastened around the subjects waist. The accelerometer axes were aligned with the vertical, mediolateral and anteriorposterior axes of the subject. The second study involved ten mixed gender recreational athletes and was conducted in a similar manner to the first. In this study, the athletes were also monitored continuously during exercise for $\mathrm{VO}_{2}$ using respiratory gas analysis from an open-circuit calometric system. Mass, height and leg length of the athletes were recorded at the time of the tests.

\section{A. Hardware}

Two dual axis, $\pm 2 \mathrm{~g}$ Analog Devices accelerometers (ADXL202E), tested as having an overload in excess of $\pm 7 \mathrm{~g}$, were mounted to the surface of a data acquisition module and aligned perpendicular to each other thereby creating a tri-axial accelerometer system. Calibration of the tri-axial accelerometer system used an inertial reference calibration technique proposed by Lai et al. [5]. The accelerometers formed part of a general-purpose sealed athlete monitoring system. This system comprised of an Hitachi H8 microcontroller based acquisition board, a low-power real time operating system [6], communications via Infra-Red circuitry and a power board with an inductive charging circuit and batteries [7]. Accelerometers were sampled at $150 \mathrm{~Hz}$ using the microcontroller's 10-bit analog-to-digital converters with data logged to an internal 32-megabyte flash memory card. At this sampling rate, the memory was sufficient for 48 hours of compressed data. The motorized treadmill and the respiratory gas open-circuit calometric system were in-house custom built systems developed by the Physiology Department at the Australian Institute of Sport (AIS).

\section{ANALYSIS PHASES}

Analysis of the collected data occurred in distinctive phases. Initial processing was performed to collect basic data and confirm the operation of the system. The second phase identified sources of individual variation. The final analysis phase identified anthropometric or biomechanical correlates of the variation in accelerometry output.

\section{A. Initial Processing}

Post-processing of logged raw data was used to: confirm the functioning of the system, identify the factors that impacted the accelerometer signal and to exploit the signal subsequently obtained. $\mathrm{VO}_{2}$ processing was performed as outlined in Saunders et al. [8].

Using a $0.9 \mathrm{~Hz}$ Hamming Windowed Finite Impulse Response (FIR-H) filter, the triaxial acceleration signal was split into orientation (low pass output) and ambulatory signals (high pass output). The ambulatory signal was used to generate $\mathrm{EE}$ estimators referred to as 'Accelerometer Counts'. Uniaxial accelerometer counts were calculated from the integral of the square of the vertical acceleration, the triaxial accelerometer counts used the integral of the sum of the squares from the triaxial acceleration. The integral was taken as a ten second average from a period of steady state walking or running at each speed. Step frequency was automatically estimated using a count of samples between successive positive zero crossings in the vertical acceleration. Step frequency was also manually calculated by counting the cycles in the vertical acceleration in the tensecond segment used for the above process.

\section{1) System Confirmation Results}

Results were in line with results from other studies, regression analysis of speed vs. EE estimates from $\mathrm{VO}_{2}$ (corrected for the subject's mass), gave a correlation coefficient of $\mathrm{R}^{2}=0.96$. Triaxial and uniaxial accelerometer counts correlated with walking speed $\left(\mathrm{R}^{2}=0.83, \mathrm{R}^{2}=0.62\right)$. Uniaxial accelerometer counts for running varied widely and gave no useful correlation with speed (Fig.2(a)). Triaxial accelerometer counts for running gave a range of correlations with substantial inter-subject variation (Figs.2(a) \& (b)). Step frequency had strong correlation with speed (individual $\mathrm{R}^{2}=0.90-1.00$, Group $\mathrm{R}^{2}=0.48$ ) (Fig.1). For individuals, step frequency also correlated highly with mass-adjusted $\mathrm{VO}_{2} \mathrm{EE}$ estimates $\left(\mathrm{R}^{2}=0.80-0.99\right)$. In contrast, the group results were weaker $\left(\mathrm{R}^{2}=0.29\right)$.

\section{B. Analysis of Sources of Variance}

From Fig.2(b), individual variations in both slope and magnitude were observed. As vertical acceleration was the predominant source of signal power, this signal was investigated. An FIR-H filter was used to remove signal above twice the step frequency, multiple left/right step pairs were averaged at each speed, and gait cycle normalization used to compare signals from different step frequencies. The output of this processing (Fig.2(c)), identified characteristics of the vertical acceleration that corresponded with the slope of the accelerometer counts of Fig.2(b). Athletes $1 \& 7$ exhibited relatively flat accelerometer counts (Fig.2(b)) and consistent vertical acceleration across all running speeds (Fig.2(c)). Athletes $4 \& 9$ exhibited both increased vertical acceleration and increased accelerometer counts as running speed increased. The slope of the Fig.2(b) regression lines was therefore an indicator of this characteristic.

Fourier analysis of the running signal identified that for most test subjects, almost the entire signal from the vertical channel was located at the step frequency. Assuming the signal was approximately sinusoidal, acceleration and

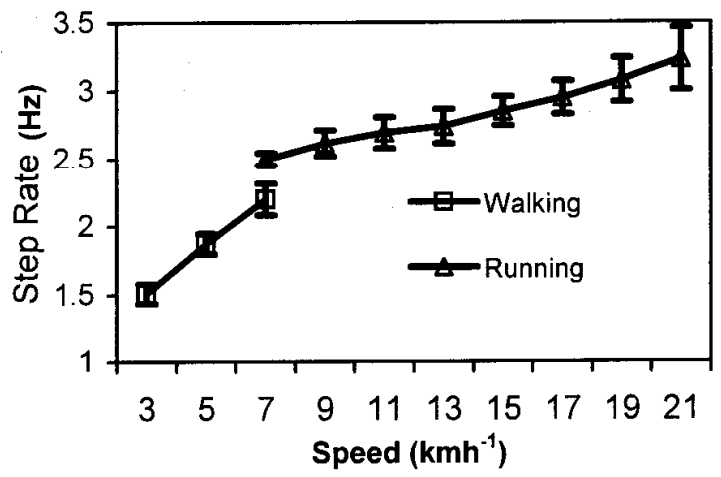

Figure 1. Step frequency for walking and running, with \pm 1 SD bars. 

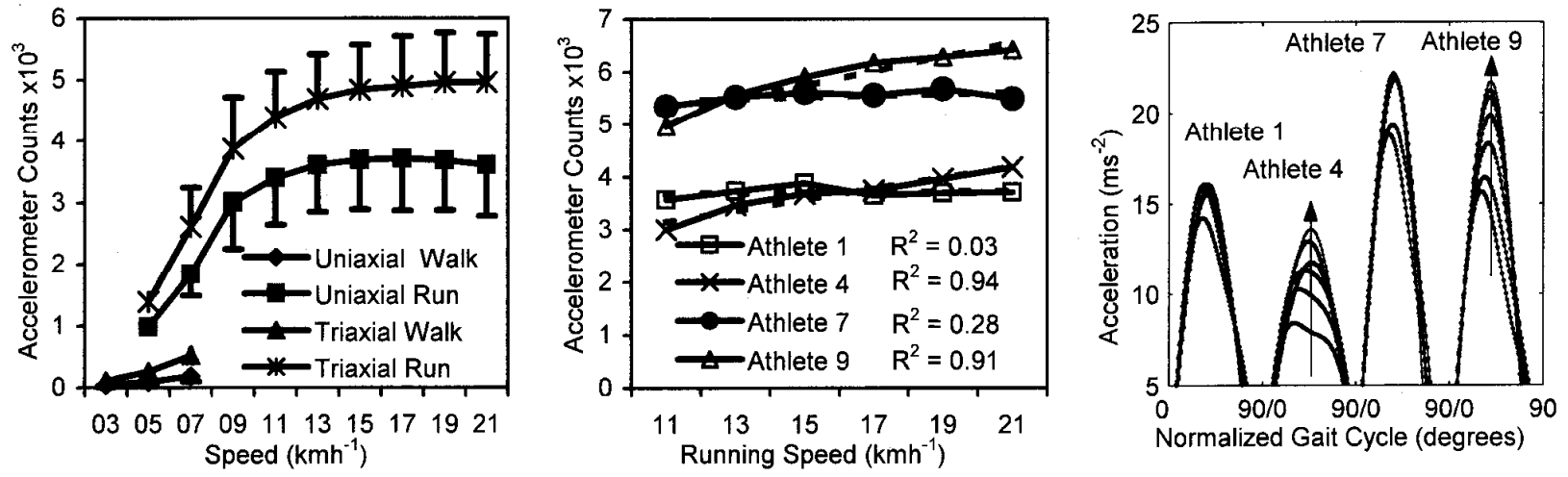

Figure 2. (a) Vertical-Uniaxial and Triaxial accelerometer counts for walking and running with \pm 1 SD bars. (b) Individual results for athletes with the highest and lowest triaxial accelerometer counts. Dashed trend lines are included in the figure. (c) Gait Cycle normalized, multi-speed, vertical acceleration signal for the four athletes in (b) (left foot only). X-axis degrees indicate the proportion of stride cycle (complete left/right step combination $=360 \mathrm{degrees)}$. The arrows indicate the change in the acceleration as running speed increased (athletes $4 \& 9$ only).

accelerometer counts were functionally related to the physical displacement of the athlete's body and the step frequency. Accelerometer counts for $9 \mathrm{kmh}^{-1}$, the lowest common running speed, were normalized using the average $9 \mathrm{kmh}^{-1}$ step frequency to give an estimated relative displacement factor (Fig.3(a)). This result indicated that the acceleration counts were proportional to a factor other than step-frequency. A comparison of the average magnitude of the $9-21 \mathrm{kmh}^{-1}$ triaxial accelerometer counts of Fig.2(b) to the $9 \mathrm{kmh}^{-1}$ step frequency appears in Fig.3(d). This indicated a strong relationship between the $9 \mathrm{kmh}^{-1}$ step frequency and the overall accelerometer count magnitude.

\section{Anthropometric and Biomechanical Analysis}

From regression analysis, body mass did not appear to affect the $9 \mathrm{kmh}^{-1}$ step frequency, relative displacement, or the slope of the accelerometer count regression line. Mass did affect the slope of the step-frequency vs. speed regression lines with a correlation coefficient of $r=0.57$.

Leg-length had a negative correlation to $9 \mathrm{kmh}^{-1}$ step frequency $(r=-0.67$, Fig.3(b)) and to the slope of the accelerometer count regression line for running $(r=-0.76)$. Conversely, leg length had a positive correlation to estimated
$9 \mathrm{kmh}^{-1}$ relative displacement $(\mathrm{r}=0.51$, Fig.3(c) $)$. Presumably, longer legs tend to reduce step-frequency and at the same time increase displacement. The overall magnitude of the triaxial acceleration counts of Fig.2(b) appeared to influenced by initial step frequency and hence leg-length.

Considering the influence of leg-length on various biomechanical factors and the effect of mass on $\mathrm{VO}_{2} \mathrm{EE}$ estimates and step-frequency, various predictor equations were tested. A simple linear predictor incorporating leglength, step-frequency and mass resulted in a strong group correlation with the $\mathrm{VO}_{2} \mathrm{EE}$ estimate. This predictor gave a group correlation coefficient for running of $\mathrm{R}^{2}=0.81$ (Fig.4.), a large improvement from $\mathrm{R}^{2}=0.29$.

\section{Summary of Physiological, Biomechanical, Anthropometric and Accelerometer Signal Analysis.}

For ambulatory activity, leg-length, mass and natural step frequency combined to generate a good sub-maximal energy estimator. As two of these factors are constants, signal processing only needs to determine step frequency. Since accelerometer counts have been shown to give a linear EE estimate for low intensity activities, a combined approach to signal processing is possible, as indicated in Table 1.
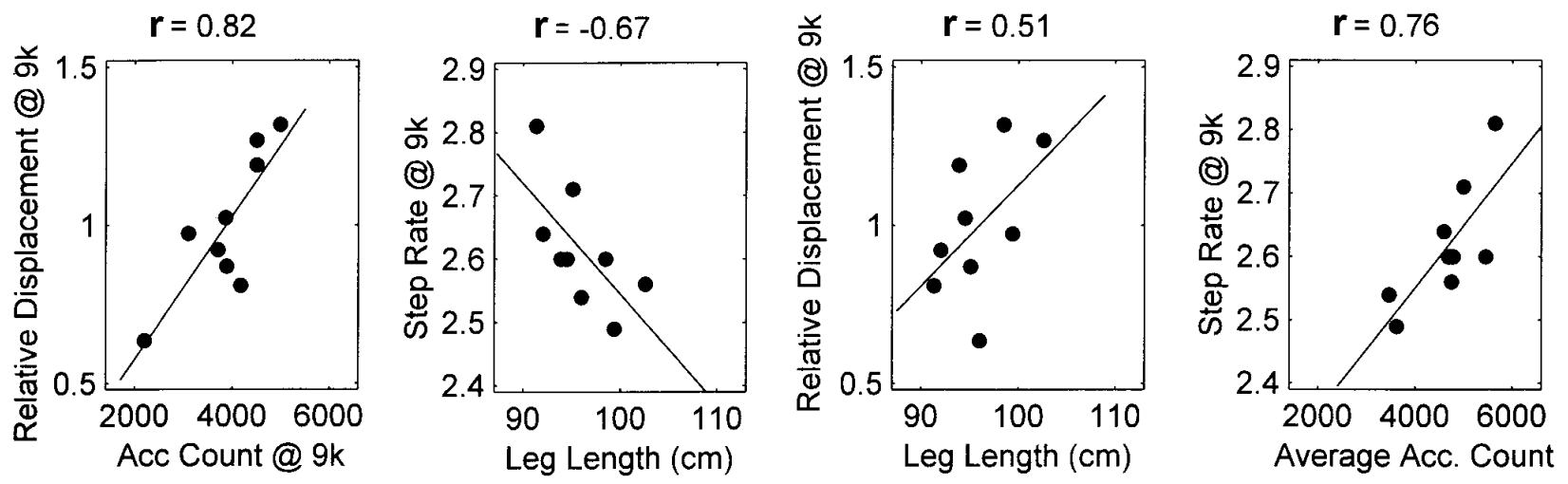

Figure 3. Scatter plots and regression analysis for: (a) relative estimated vertical displacements at $9 \mathrm{kmh}^{-1}$ (after normalising for step frequency) vs. accelerometer counts, (b) step frequency at $9 \mathrm{kmh}^{-1} \mathrm{vs}$. leg-length, (c) relative vertical displacement at $9 \mathrm{kmh}^{-1}$ (after normalising for step frequency) vs. leglength and (d) step frequency at $9 \mathrm{kmh}^{-1}$ vs average running accelerometer counts. 


\section{EMBEDded System Signal Processing}

While the foregoing discussion deals specifically with extracting the energy estimator, the total system incorporates additional functionality such as contact/tackle and orientation information. Orientation was derived using a comparison of the output of the $0.9 \mathrm{~Hz}$ low pass filter and the 'steady state' orientation generated from a long duration first order lag function. Accelerometer counts in conjunction with athlete orientation indicate activities such as jumping, hard contact, and player on the ground. This processing is an extension of the table driven approach and is indicated in Table 1.

To reduce processing load, less computationally intensive filters at lower sampling rates were assessed with outputs compared to those previously obtained. Step frequency calculation was modified to perform linear interpolation to determine zero crossing timing.

TABLE I. ENERGY ESTIMATION AND ACTIVITY CLASSIFICATION

\begin{tabular}{|l|l|l|l|l|}
\hline \multicolumn{1}{c}{$\begin{array}{c}\text { Triaxial } \\
\text { Accelerometer } \\
\text { Counts }\end{array}$} & \multicolumn{4}{|c|}{ Activity Type } \\
\cline { 2 - 5 } & Stationary & Walking & Running & Other $^{\boldsymbol{a}}$ \\
\hline Below walking band & $\mathbf{X}$ & & $\begin{array}{l}\text { Check } \\
\text { angle }^{\mathrm{b}}\end{array}$ \\
\hline Walking Band & & $\begin{array}{l}\text { Use Acc. } \\
\text { Counts }\end{array}$ & \\
\hline Running Band & & $\begin{array}{l}\text { Measure } \\
\text { Step } \\
\text { Frequency }\end{array}$ & \\
\hline Above Running Band & & & $\begin{array}{l}\text { Check } \\
\text { angle }\end{array}$ \\
\hline
\end{tabular}

a. Other includes various football code specific activities such as tackles, marks, jumping etc. b. Orientation combines with activity intensity to determine activity type.

\section{A. Complete Embedded Processing System}

The complete EE estimate signal processing, comprises three accelerometers sampled at $24 \mathrm{~Hz}$ with data stored in a three-channel circular buffer. The buffer holds approximately $1.9 \mathrm{~s}$ of data and forms the basis of a rectangular FIR orientation filter (FIR-R). Processing occurs with an inbuilt $0.9 \mathrm{~s}$ latency. The delayed triaxial sample value is used to generate or detect: (1) steady state triaxial orientation using a long duration first order lag, (2) current dynamic acceleration by subtracting the orientation filter value, (3) triaxial accelerometer counts and (4) negative to positive zero crossings on the vertical axis.

Accelerometer counts are summed in blocks and compared to threshold values stored in a table. Angle is monitored on the vertical channel by comparing the FIR-R output to the steady state first order lag output. Angles are only calculated if the accelerometer counts or the vertical orientation signal exceeds preset thresholds. Step-frequency is calculated if indicated by the triaxial accelerometer counts.

\section{B. Results}

With no comparable system performing the same analysis, results have been assessed using comparisons to manually categorized football data, video data, and treadmill and track data. Generated step frequencies from different

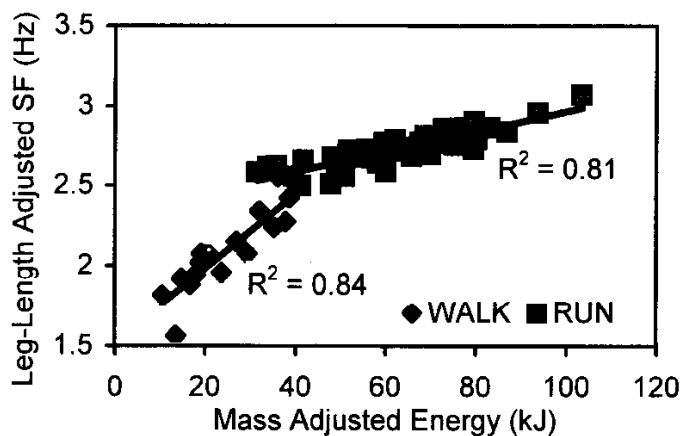

Figure 4. Normalized leg-length adjusted step rate vs. normalized mass adiusted energy. with correlation coefficients and trend lines.

activities matched those calculated manually. Discrepancies between system output and manually categorized data were identified and attributed to a variety of operator related causes.

This signal processing is considerably less intensive than the original system allowing the operating frequency of the microprocessor to be dropped to a few hundred kilohertz with a subsequent reduction in current draw.

\section{CONCLUSION}

The investigation and analysis of the variance of accelerometer-count based energy estimates identified mechanical, biomechanical and anthropometrical influences. By modifying the signal processing, EE estimators can be collected at lower cost with a higher immunity to detrimental influences and which, when combined with anthropometric measures, greatly improve the EE estimates gathered.

\section{REFERENCES}

[1] H.J. Montoye, H.C.G. Kemper, W.H.M. Saris, and R.A. Washburn, "Measuring physical activity and energy expenditure", Human Kinetics, Champaign, IL: , 1996, pp. 34-41.

[2] B.E. Ainsworth, et al., "Compendium of Physical Activities: classification of energy costs of human physical activities", Med. Sci. Sports Exerc., Vol. 25, No.1, pp71-80, 1993.

[3] H.J. Montoye, et al., "Estimation of energy expenditure by a portable accelerometer", Med. Sci. Sports Exerc. 15:403-407, 1983.

[4] G.A. King, N. Torres, C. Potter, T.J. Brooks, and K. J. Coleman, "Comparison of activity monitors to estimate energy cost of treadmill exercise", Med. Sci. Sports Exerc., Vol. 36, No. 7, pp. 1244-1251, 2004.

[5] A. Lai, D.A. James, J.P. Hayes, E.C. Harvey, "Semi-automatic calibration technique using six inertial frames of reference", Proceedings of the International Society of Optical Engineering. SPIE Vol. 5274, pp.531-542, Perth, Australia, Dec 2003.

[6] A. Wixted, D. James, D. Thiel, "Low power operating system and wireless networking for a real time sensor network", Proceedings IEEE ICITA, paper 112 , Bathurst, Australia, Nov 2002

[7] D.A. James, N. Davey, T. Rice, "An accelerometer based sensor platform for insitu elite athlete performance analysis", Proceedings IEEE Sensors conference. Vienna, October 2004.

[8] P.U. Saunders, R.D. Telford, D.B. Pyne, R.B. Cunningham, C.J. Gore, A.G. Hahn, and J.A. Hawley, "Improved running economy in elite runners after 20 days of simulated moderate-altitude exposure", Journal Appl Physiol 96: pp.931-937, 2004. 